\title{
Upgrade of Imaging Bolometers on LHD
}

\author{
Byron J. PETERSON, Evgeny A. DRAPIKO, Dongcheol SEO ${ }^{1)}$ and Naoko ASHIKAWA \\ National Institute for Fusion Science, 322-6 Oroshi-cho, Toki-shi, Gifu-ken 509-5292, Japan \\ 1) National Fusion Research Institute, Daejeon 305-806 Republic of Korea
}

(Received 10 December 2009 / Accepted 15 May 2010)

\begin{abstract}
Infrared Imaging Video Bolometers (IRVB) provide an image of the plasma radiation by using a thin metal foil to absorb the radiation, which is then imaged by an IR camera. In LHD IRVBs at Ports 6-T and 1-O have been upgraded. Both of the $\sim 1$ micron $\times 70 \mathrm{~mm} \times 90 \mathrm{~mm}$ gold foils have been replaced with $\sim 2.5$ micron Pt foils of the same area for greater sensitivity (Pt vs $\mathrm{Au}$ ) and absorption of higher energy photons estimated up to $8.2 \mathrm{keV}$. Also the IR cameras have been replaced; an FLIR/SC500 $(\sim 100 \mathrm{mK}, 60 \mathrm{fps}, 320 \times 240$ pixels $)$ IR camera at Port 1-O with a FLIR/Phoenix $(\sim 16 \mathrm{mK}, 345 \mathrm{fps}, 320 \times 256$ pixels $)$ IR camera at Port 10-O and an AGEMA/THV 900 LW $(\sim 190 \mathrm{mK}, 15 \mathrm{fps}, 136 \times 272$ pixels $)]$ with a FLIR SC-4000 $(\sim 19 \mathrm{mK}, 327 \mathrm{fps}, 320 \times 256$ pixels $)$ IR camera at port 6-T. In the current campaign these IRVBs will use $8 \mathrm{~mm} \times 8 \mathrm{~mm}$ apertures resulting in $12 \times 16$ channels on the foil. The noise equivalent power density for these two new IRVBs ranges from $\sim 360 \mu \mathrm{W} / \mathrm{cm}^{2}$ for $327 \mathrm{fps}$ operation to $3.6 \mu \mathrm{W} / \mathrm{cm}^{2}$ for $15 \mathrm{fps}$ operation, which is over two orders of magnitude of improvement over the previous IRVB at Port 6-T.
\end{abstract}

(c) 2010 The Japan Society of Plasma Science and Nuclear Fusion Research

Keywords: bolometer, infrared, LHD, radiation, plasma

DOI: $10.1585 /$ pfr.5.S2095

\section{Introduction}

Bolometers play an important role in diagnosing radiation losses from the plasma. Usually resistive bolometers have been used to fulfill this role in magnetic plasma confinement experiments. Typically these are arrayed around one poloidal cross-section to give a two dimensional (2D) radiation profile through a tomographic reconstruction of the line averaged signals from each detector [1]. This is sufficient to measure the total radiated power in a tokamak by assuming toroidal symmetry. However, in nonaxisymmetric configurations such as the Large Helical Device (LHD) such an assumption is not possible. This has motivated the development of a new type of bolometer detector called the InfraRed imaging Video Bolometer (IRVB) [2-5]. This diagnostic provides an image of the plasma radiation which is divided up into a 2D array of detectors. The large numbers of channels available from this diagnostic are useful for determining the threedimensional radiation source distribution, especially when the IRVB has a tangential view of the plasma. The IRVB consists of a thin metal foil which absorbs the plasma radiation incident on its front side through an aperture. The back side of the foil is blackened to reradiate the absorbed radiation in the infrared (IR) which temperature is then measured by an IR camera placed outside the vacuum chamber. The 2D temperature distribution on the foil is used to solve the $2 \mathrm{D}$ heat diffusion equation for the $2 \mathrm{D}$ radiated power incident on the foil from the plasma. The collimation of the radiation through the aperture enables

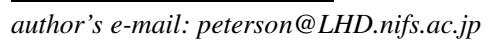

the $2 \mathrm{D}$ radiation profile on the foil to serve as an image of the plasma radiation. The calibration of the foil involves the determination of the $2 \mathrm{D}$ distribution of thermo-physical properties such as thermal diffusivity, thermal conductivity, foil thickness and blackbody emissivity [6]. In this paper two IRVBs which have been recently upgraded on LHD are described.

\section{Sensitivity of the IRVB}

The sensitivity of the IRVB is given by Eq. 10 in Ref. 3 which is reproduced here as the noise equivalent power density (NEPD)

$$
\frac{\eta_{\mathrm{IRVB}}}{l^{2}}=\frac{\sqrt{10} k t_{\mathrm{f}} \sigma_{\mathrm{IR}}}{l^{2} \sqrt{m N}} \sqrt{1+\frac{l^{4}}{5 \kappa^{2} m^{2} \Delta t_{\mathrm{IR}}^{2}}}
$$

in terms of the thermal conductivity of the foil, $k$, the thickness of the foil, $t_{\mathrm{f}}$, the sensitivity of the IR camera (noise equivalent temperature), $\sigma_{\mathrm{IR}}$, the time resolution of the IR camera, $\Delta t_{\mathrm{IR}}$, the number of time frames averaged over, $m$, the number of IR pixels averaged over to get one bolometer pixel, $N$, the dimension of the bolometer pixel, $l$, and the thermal diffusivity of the foil, $\kappa$. The black-body radiation term is neglected since it does not play a role at room temperature where the LHD IRVBs operate. From this equation one can see that the sensitivity is a function of many parameters. Sensitivity can be improved by averaging over more IR camera frames and IR camera pixels at the expense of time resolution and number of bolometer pixels (spatial resolution), respectively. The time resolution is ultimately limited by the IR camera frame rate, but 


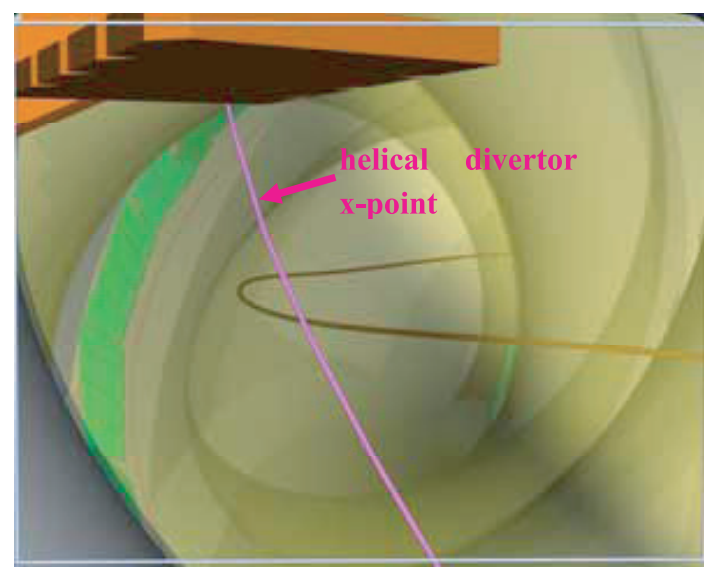

Fig. 1 Field of view of IRVB at LHD Port 6-T.

also by the thermal time of the foil which is described by the right hand ratio under the radical in Eq. 1, which comes from the time derivative term of the heat diffusion equation for the foil. Therefore if the time resolution of the imaging bolometer is much faster than the thermal time of the foil, then the sensitivity will be decreased. Sensitivity improves as the foil becomes thinner, but a thinner foil means that the upper limit of photon energy that can be measured is reduced as is explained in Section 5. Also sensitivity is a function of the foil material thermal properties $k$ and $\kappa$.

\section{IRVB at Port 6-T}

The IRVB at port 6-T previously used an AGEMA/THV 900 LW $(\sim 190 \mathrm{mK}, 15 \mathrm{fps}, 136 \times 272$ pixels) IR camera [7]. This has been replaced with a FLIR SC-4000 ( 18 mK, $327 \mathrm{fps}, 320 \times 256$ pixels $)$ IR camera. The foil previously was a $\sim 1$ micron gold foil and has been replaced with $\mathrm{a} \sim 2.5$ micron $\mathrm{Pt}$ foil. The foil size is $9 \mathrm{~cm} \times 7 \mathrm{~cm}$. The aperture is $8 \mathrm{~mm} \times 8 \mathrm{~mm}$ resulting in a $16 \times 12$ pixel IRVB whose field of view is shown in Figure 1. Sample temperature variation images $(18 \times$ 14 pixels $)$ and radiation power density images $(16 \times 12$ pixels) are shown in Figures 2 and 3 respectively. The radiated power density is calculated from the temperature variation using a numerical scheme to solve the heat diffusion equation in the foil [3]. The noise equivalent temperature (NET) is measured to be $18.5 \mathrm{mK}$ for an IR camera integration time of $2 \mathrm{~ms}$. In terms of temperature we have a signal to noise ratio $(\mathrm{S} / \mathrm{N})$ of $\sim 210$. Using Eq. 1 the NEPD is calculated as $0.36 \mathrm{~mW} / \mathrm{cm}^{2}$ giving a $\mathrm{S} / \mathrm{N}$ $\sim 11$. If we degrade the time resolution from $\sim 3 \mathrm{~ms}$ to $16.6 \mathrm{~ms}$ ( $60 \mathrm{fps}$ ) then the NEPD is reduced to $28 \mu \mathrm{W} / \mathrm{cm}^{2}$. In that case we can also increase the IR camera integration time from $2 \mathrm{~ms}$ to $16 \mathrm{~ms}$ which will increase the IR camera signal by a factor of 8 and improve the $S / N$. If we degrade the time resolution further to $67 \mathrm{~ms}(15 \mathrm{fps})$, which is the same as the previous IRVB, then the NEPD drops to $3.6 \mu \mathrm{W} / \mathrm{cm}^{2}$ Even though it has slightly more pixels and a thicker foil, the new IRVB at Port 6-T is 140 times more
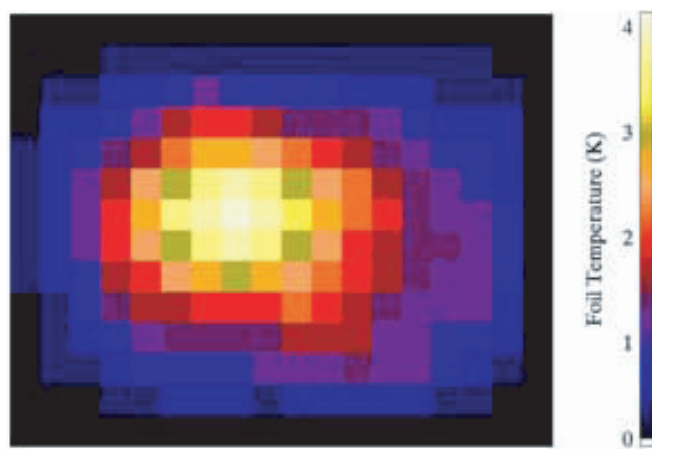

Fig. 2 Sample temperature increase data from IRVB at LHD Port 6-T (shot 97279).
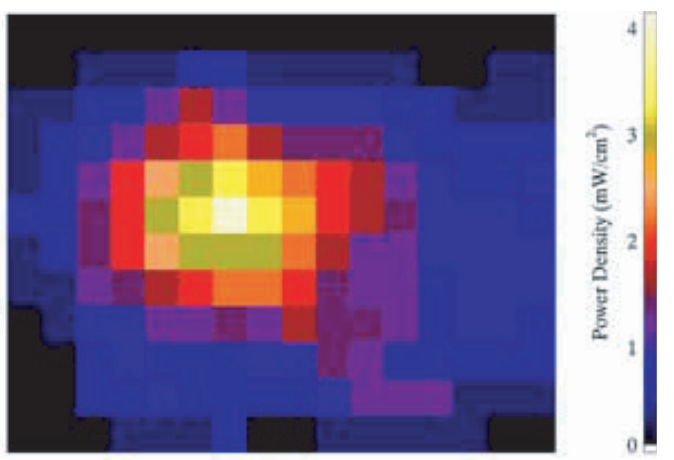

Fig. 3 Sample radiated power density data from IRVB at LHD Port 6-T (shot 97279).

sensitive than the previous IRVB $\left(500 \mu \mathrm{W} / \mathrm{cm}^{2}\right)$ at Port 6-T [7].

\section{IRVB at Port 10-O}

The IRVB at port 10-O previously was installed at Port 1-O and used an FLIR/SC500 ( 100 mK, $60 \mathrm{fps}, 320$ $\times 240$ pixels) IR camera. According to Eq. 1 , the NEPD of this IRVB is $0.127 \mathrm{~mW} / \mathrm{cm}^{2}$. This has been replaced with a FLIR/Phoenix $(\sim 11 \mathrm{mK}, 345 \mathrm{fps}, 320 \times 256$ pixels $)$ IR camera. The foil previously was a $\sim 1$ micron gold foil and has been replaced with a $\sim 2.5$ micron Pt foil. The foil size is $9 \mathrm{~cm} \times 7 \mathrm{~cm}$. The aperture is $8 \mathrm{~mm} \times 8 \mathrm{~mm}$ resulting in a $16 \times 12$ pixel IRVB whose field of view is shown in Figure 4. Sample temperature variation data $(18 \times 14$ pixels) and radiation power density data $(16 \times 12$ pixels $)$ are shown in Figures 5 and 6 respectively. The NET is measured to be $15.8 \mathrm{mK}$ for an integration time of $2 \mathrm{~ms}$. In terms of temperature we have an $\mathrm{S} / \mathrm{N} \sim 170$. Using Eq. 1 the NEPD is calculated as $0.32 \mathrm{~mW} / \mathrm{cm}^{2}$ giving an $\mathrm{S} / \mathrm{N} \sim 10$. If we degrade the time resolution from $\sim 3 \mathrm{~ms}$ to $16.6 \mathrm{~ms}$ ( $60 \mathrm{fps}$ ), which is equivalent to that of the previous SC500 camera, then the NEPD is reduced to $23.5 \mu \mathrm{W} / \mathrm{cm}^{2}$. This is 5.4 times more sensitive than the previous IRVB at port 1-O. A comparison of the sensitivity of the 4 IRVBs in terms of the time resolution $\left(\Delta t_{\mathrm{IRVB}}=\mathrm{m} \Delta t_{\mathrm{IR}}\right)$ and the number of frames averaged over, $\mathrm{m}$, is shown in Figure 7. 


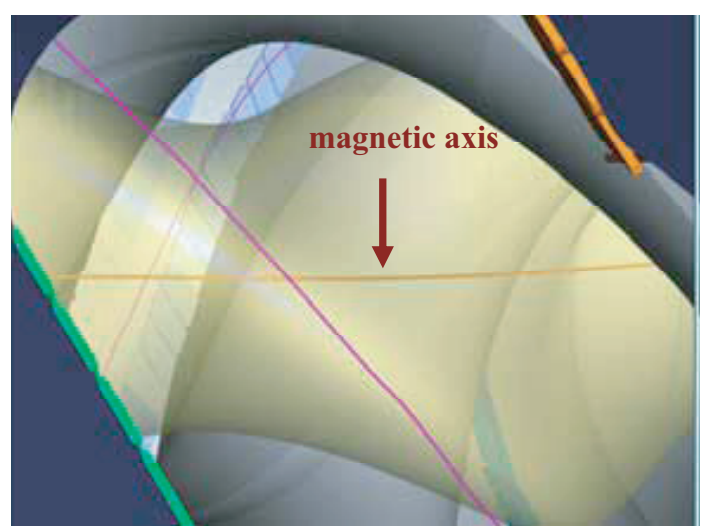

Fig. 4 Field of view of IRVB at LHD Port 10-O.
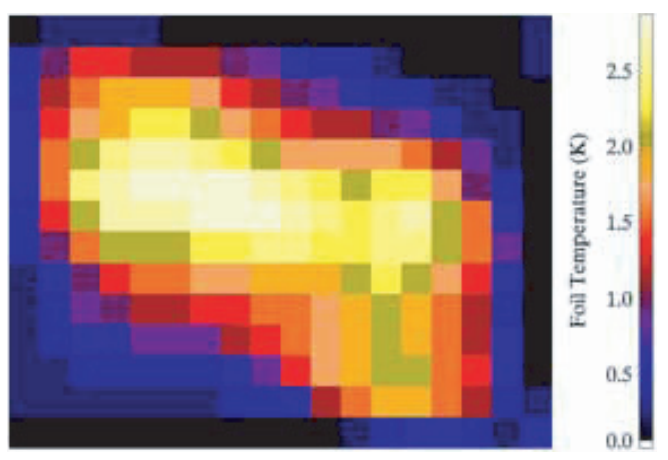

Fig. 5 Sample temperature increase data from IRVB at LHD Port 10-O (shot 97279).
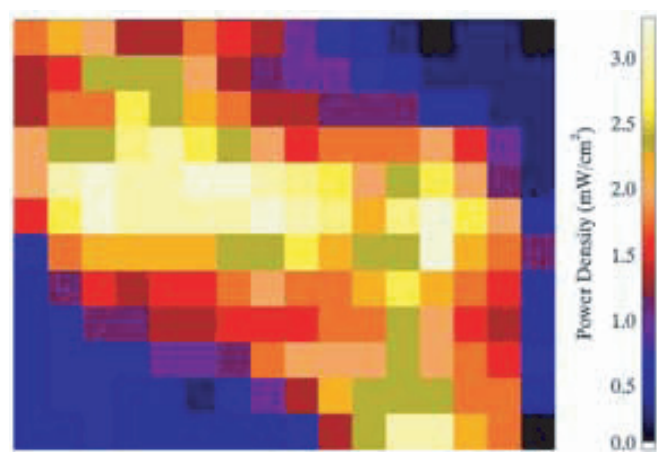

Fig. 6 Sample radiated power density image from IRVB at LHD Port 10-O (shot 97279).

\section{Photon Energy Response}

By changing the foils from 1 micron gold to $2.5 \mathrm{mi}-$ cron platinum we increase the estimated upper limit of absorbed photons from 5.5 to $8.2 \mathrm{keV}$ as can be seen from the calculated attenuation length as a function of photon energy for $\mathrm{Au}$ and $\mathrm{Pt}$ in Figures 8a and b, respectively.

\section{Conclusions}

Two imaging bolometers have been upgraded on LHD. This upgrade of the IR cameras has resulted in

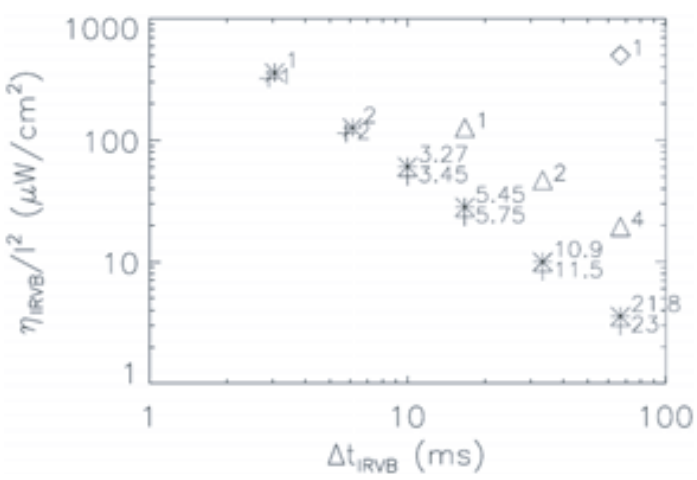

Fig. 7 Sensitivity versus time resolution for IRVBs at 6-T (diamond - former, * - upgrade) and 10-O (triangle - former, +- upgrade) for different $m$ values (shown next to symbols in plot).
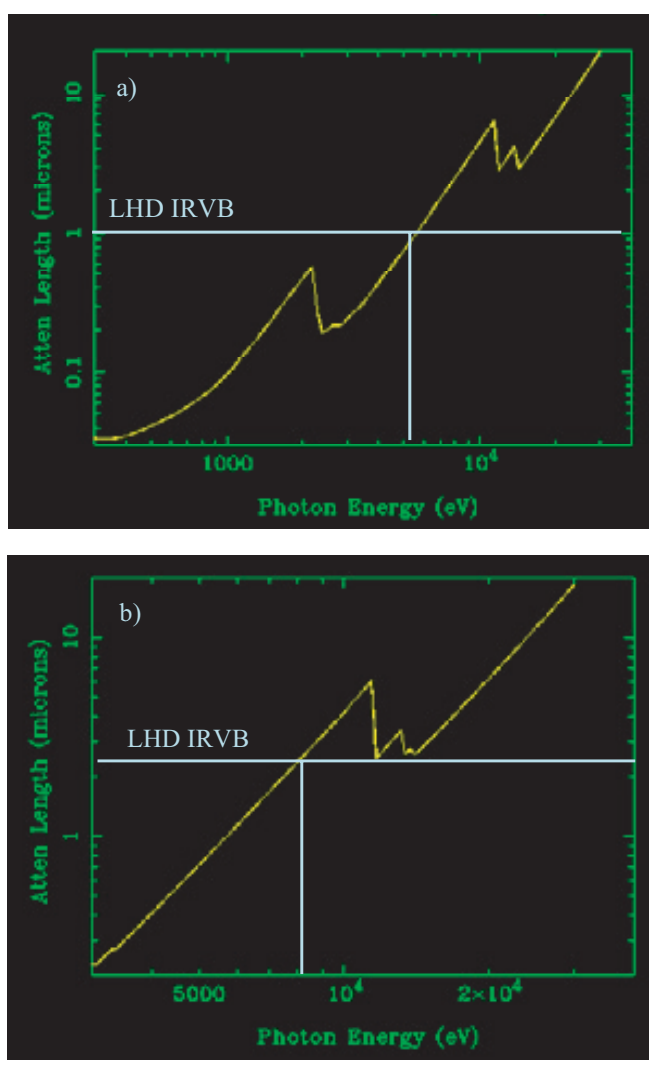

Fig. 8 Attenuation length versus photon energy in a) Au and b) Pt [8].

higher sensitivity and faster frame rates. The improvement in sensitivity allowed the use of a thicker foil, thereby expanding the estimated upper limit of measurable photon energies. The expanded photon energy range will help to better diagnose higher energy photons from the plasma core such as bremstrahlung that has accompanied higher energy plasmas recently. Faster frame rates up to 345 frames per second will allow the diagnosis of faster phenomena, but at lower sensitivity. Preliminary data has been acquired. 
Starting in the next experimental campaign the following changes are anticipated. (1) Taking advantage of the improved sensitivity, aperture sizes will be reduced to increase the number of bolometer pixels and the spatial resolution. (2) Triggering will be done with the LHD trigger and data acquisition and analysis will be automated. (3) An additional imaging bolometer will be brought into operation at Port 6.5-U. (4) Foil calibration will be improved using a refurbished calibration chamber and the three foils will be recalibrated. (5) Using the data from 3 IRVBs initial efforts at 3-D tomography will be made in LHD. Research targets include detached plasmas, radiative collapse, impurity hole, and other radiative phenomena in LHD.

[1] A. W. Leonard et al., Rev. Sci. Instrum. 66, 1201 (1995).

[2] B. J. Peterson, Rev. Sci. Instrum. 71, 3696 (2000).

[3] B. J. Peterson et al., Rev. Sci. Instrum. 74, 2040 (2003).

[4] B. J. Peterson et al., Rev. Sci. Instrum. 79, 10E301 (2008).

[5] B. J. Peterson et al., Plasma Fusion Res. 2, S1018 (2007).

[6] H. Parchamy et al., Rev. Sci. Instrum. 77, 10E515 (2006).

[7] B. J. Peterson et al., Rev. Sci. Instrum. 72, 923 (2001).

[8] Lawrence Berkeley Laboratory: http://www-cxro.lbl.gov/ optical_constants/atten2.html 